\title{
Acute Endolymphatic Hydrops Generated by Exposure of the Ear to Nontraumatic Low-Frequency Tones
}

\author{
Alec N. Salt \\ Department of Otolaryngology, Washington University School of Medicine, St. Louis, MO 63110, USA
}

Received: 18 August 2003; Accepted: 18 December 2003; Online publication: 4 May 2004

\section{ABSTRACT}

Low-frequency sounds presented at high nontraumatizing levels induce temporary hyperacusis in humans and animals. One explanation of this finding is that the basilar membrane operating point may be disturbed by an endolymph volume change. This possibility was investigated using volume and flow markers iontophoresed into the endolymphatic space of guinea pigs. Marker concentrations were measured with ion-selective microelectrodes placed apically and basally to the iontophoresis site during exposure of the ear to low-frequency tones. Concentration changes were interpreted quantitatively using a finiteelement model of the endolymphatic space that allowed changes of endolymph cross-sectional area and flow to be derived. Stimulation with a $200 \mathrm{~Hz}$ tone at $115 \mathrm{~dB}$ SPL for 3 min produced marker concentration changes consistent with the induction of transient endolymphatic hydrops and a basally directed displacement of endolymph. Endocochlear potentials were greater than normal after the exposure when hydrops was present. During identical tone exposures of animals without marker, we found that action potential (AP) threshold changes and endolymph potassium changes associated with the hydropic state were small. Marker concentration changes were compared with changes in endocochlear potential and AP thresholds for a range of exposure frequencies and levels. AP hypersensitivity occurred with $200 \mathrm{~Hz}$ exposure levels below those inducing endolymph volume disturbances. Endolymph volume changes are thought to be the result of, rather than

Correspondence to: Alec N. Salt • Department of Otoloryngology, Box 8115 - Washington University School of Medicine -660 South Euclid Avenue - St. Louis, MO, 63110 • Telephone (314) 362-7560; Fax: (314) 362-7522; email: salta@wustl.edu the cause of, changes in operating point of the cochlear transducer. The observations that auditory threshold and endolymph potassium changes are minimal under conditions where substantial endolymphatic hydrops is present is relevant to our understanding of the hearing loss in patients with Ménière's disease.

Keywords: endolymph volume, Ménière's disease

\section{INTRODUCTION}

The ionic composition of endolymph is remarkably stable during most types of sound stimulation of the ear. Salt and Konishi (1979) reported that stimulation of guinea pigs with wideband noise at levels up to $115 \mathrm{~dB}$ produced only minor increases of endocochlear potential (EP) and endolymph $\mathrm{K}^{+}$, even though the $115 \mathrm{~dB}$ level produced a substantial impairment of cochlear sensitivity. Only when extremely high sound levels are delivered do substantial changes of endolymph $\mathrm{K}^{+}$occur. Melichar and Syka (1980) showed that $142 \mathrm{~dB}$ octave band noise presented to guinea pigs caused dramatic reductions in both EP and endolymph $\mathrm{K}^{+}$, which recovered over a 20-day period. Salt and Konishi (1979) similarly found that wideband noise delivered at $125 \mathrm{~dB}$ SPL caused large reductions of EP and endolymph $\mathrm{K}^{+}$.

In contrast, low-frequency tones appear to produce unusual effects on the ear, causing a transient enhancement of hearing sensitivity immediately postexposure. This was initially reported in humans by Hirsh and Ward (1952), who called it the "two-minute bounce.' Kemp (1982) subsequently observed an 
increase in the amplitude of transient otoacoustic emissions following low-frequency tone exposures. The emission increase peaked at 1-1.5 min after the tone ended, which he also referred to as a "bounce." The origins of the enhancements of auditory sensitivity and acoustic emissions after low-frequency tones were further investigated by Kirk and Patuzzi (1997) and Kirk et al. (1997). Using measurements of cochlear microphonics and otoacoustic emissions, Kirk and Patuzzi (1997) confirmed that the transient hypersensitivity seen approximately $1 \mathrm{~min}$ after lowfrequency tone exposure was cochlear, rather than neural, in origin. Based on an analysis of the cochlear microphonic waveform from which the operating point of the cochlear transducer was derived, they suggested that the sensitivity changes were caused by a shift in the operating point of the cochlear transducer comparable to a displacement of the organ of Corti towards scala tympani (Kirk et al. 1997). One explanation put forward to account for this shift was through an osmotic mechanism, in which the lowfrequency sound caused a transient "scala media bloating." The concept that low-frequency tones influence the endolymphatic system was further supported by an in vitro study by Flock and Flock (2000). In that study, isolated cochleae were maintained by high-rate perilymphatic perfusion while tissue boundaries in the apical turn were visualized directly with a confocal microscope. Stimulation with $140 \mathrm{~Hz}$ tone bursts between 90 and $115 \mathrm{~dB}$ was found to produce substantial endolymphatic hydrops in the apical turn, which rapidly recovered to normal after cessation of the tone.

In the present study, we have investigated the endolymph volume and functional changes produced by low-frequency tones. This was performed using ionic markers, iontophoresed into endolymph without volume disturbance and monitored with ionselective electrodes during experimental manipulations. These markers have been used previously to quantify endolymph cross-sectional area and flow in normal ears (Salt et al. 1986), in ears with chronic endolymphatic hydrops (Salt et al. 1995), and in ears in which endolymph was manipulated acutely (Salt and DeMott 1997, 1998).

\section{METHODS}

Pigmented NIH strain guinea pigs weighing 300$500 \mathrm{~g}$ were anesthetized with Inactin (sodium thiobutabarbital) at a dose of $100 \mathrm{mg} / \mathrm{kg}$ given intraperitoneally. The trachea was cannulated and the animal was mechanically ventilated, maintaining an end-tidal $\mathrm{CO}_{2}$ level of $4 \%-5 \%$. Body temperature was maintained at $39^{\circ} \mathrm{C}$ with a thermistor-controlled heating pad. An intravenous line was placed in the external jugular vein for anesthetic supplements. The inner ear on the right side was exposed by a ventrolateral approach and the bulla was opened widely to gain access to the cochlea, leaving middle ear structures intact. Prior to recordings a muscle relaxant, pancuronium bromide (IV, to effect), was given to suppress movement artifacts and middle ear muscle effects. The results of this study are based on findings in 29 guinea pigs.

Sounds were delivered through a hollow ear bar inserted into the sectioned ear canal. One of two sound sources was used, according to the needs of the experiment. For those experiments requiring $8 \mathrm{kHz}$ stimuli for threshold testing, an Etymotic ER-2 driver was used. In other experiments, such as those requiring levels up to $125 \mathrm{~dB}$ SPL at $200 \mathrm{~Hz}$, a Sennheiser HD 580 driver coupled to the closed cavity was used. Sound stimuli were calibrated using a B\&K 4135 microphone with a probe tube adaptor that terminated at the tip of the earbar. An automated routine was used to track a specified sound level (typically $75 \mathrm{~dB}$ SPL) in the ear canal as frequency was varied in 0.25 octave steps. All aspects of sound stimuli delivered (frequency, level, and timing) were computer controlled.

Evoked potentials were recorded from an $\mathrm{Ag} / \mathrm{AgCl}$ ball electrode placed on the round window membrane, recorded differentially relative to a platinum needle inserted under the skin at the vertex. The signal was amplified $5000 \times$ and filtered below $30 \mathrm{~Hz}$ and above $30 \mathrm{kHz}$ with a Princeton PAR113 amplifier. Waveforms were digitized and averaged using an Analogic D6000 under computer control. Cochlear action potential (AP) responses were collected by averaging responses to 10 condensation onset and 10 rarefaction onset (inverted) tone burst stimuli, thereby canceling the cochlear microphonic component. AP thresholds were established in an automated routine using an amplitude criterion of $10 \mu \mathrm{V}$, which was approximately 4 times greater than the recording noise level with no stimulus. Stimuli were increased in $5 \mathrm{~dB}$ steps until AP amplitude exceeded the criterion, and then decreased in $5 \mathrm{~dB}$ steps until the AP amplitude was below the criterion. The threshold value was established by interpolation between above- and below-threshold response amplitudes, at the point corresponding to $10 \mu \mathrm{V}$ amplitude. In experiments where cochlear sensitivity was monitored during experimental treatments, AP thresholds at two frequencies were determined and the measurements repeated sequentially at $40 \mathrm{~s}$ intervals.

Measurement of flow and cross-sectional area of endolymph were performed using the ionic markers tetramethylammonium $\left(\mathrm{TMA}^{+}\right)$or hexafluoroarsenate $\left(\mathrm{AsF}_{6}{ }^{-}\right)$. Markers were iontophoresed into 
endolymph of the second cochlear turn from glass electrodes filled with a $500 \mathrm{mM}$ electrolyte solution (TMACl or $\mathrm{LiAsF}_{6}$, respectively). The electrode tips were filled with $0.5 \%$ agarose gel to prevent volume movement of the electrolyte. Ions were retained with a current of $-10 \mathrm{nA}$ and ejected with a continuous, nonpulsed current of $50 \mathrm{nA}$ controlled by a WPI 260 current generator. The second turn location is approximately $11 \mathrm{~mm}$ from the base measured along the endolymphatic space and has a best frequency of approximately $3 \mathrm{kHz}$ (Greenwood 1990). Marker concentration in endolymph was monitored by 1 or 2 ion-selective microelectrodes inserted into endolymph of the same turn a small distance (nominally $0.5 \mathrm{~mm}$ ) from the injection site. The placement of electrodes is shown schematically in Figure 1. The terms "basal" and "apical" refer to their placement with respect to the injection site along the uncoiled endolymphatic space. In each experiment the distance between the iontophoretic and ion-selective electrodes was measured using a calibrated reticule in the operating microscope and data analysis was based on the actual distances. Ion electrodes were made from double-barreled glass pipettes, with one barrel silanized by exposure to dimethyldichlorosilane vapor. For $\mathrm{K}^{+}$and $\mathrm{TMA}^{+}$selective electrodes, the nonsilanized barrel was filled with $500 \mathrm{mM}$ $\mathrm{NaCl}$, and for $\mathrm{AsF}_{6}{ }^{-}$-selective electrodes, it was filled with $500 \mathrm{mM} \mathrm{KCl}$. This barrel served both as a reference for the ion electrode and for the recording of EP. The silanized barrel was filled with $500 \mathrm{mM}$ $\mathrm{KCl}$ and a small column of ion exchanger was drawn into the tip. For $\mathrm{K}^{+}$-selective electrodes the exchanger was Fluka 60398 (Fluka, Ronkonkoma, NY). For $\mathrm{TMA}^{+}$-selective electrodes the exchanger consisted of a $5 \%$ solution of potassium tetrakis (4chlorophenyl) borate in 2-nitrophenyloctylether (Fluka). For $\mathrm{AsF}_{6}{ }^{-}$-selective electrodes, the exchanger was $10 \mathrm{mM}$ crystal violet dye (Sigma, St. Louis, MO) dissolved in 3-nitro-o-xylene (Fluka). $\mathrm{TMA}^{+}$- and $\mathrm{AsF}_{6}{ }^{-}$-selective electrodes were calibrated in 5 standards at body temperature containing 0,80 , 160,640 , and $1280 \mu \mathrm{M}$ TMACl or $0,10,100$ and $1000 \mu \mathrm{M} \mathrm{LiAsF}$, respectively, in a background of $150 \mathrm{mM} \mathrm{KCl}$. Nonlinear calibration curves were fitted to the data points based on the sensitivity of a test ion in the presence of an interfering background ion (Salt and Vora 1991), which allowed accurate resolution of low tracer concentrations. $\mathrm{K}^{+}$selective electrodes were calibrated in 3 standards containing 100,150 , or $200 \mathrm{mM} \mathrm{KCl}$, with a straight line fitted to the plot voltage versus the logarithm of $\mathrm{K}^{+}$concentration used to derive concentration values. In all ionic measurement experiments, ion concentration and EP data were sampled and stored at $10 \mathrm{~s}$ intervals under computer control.

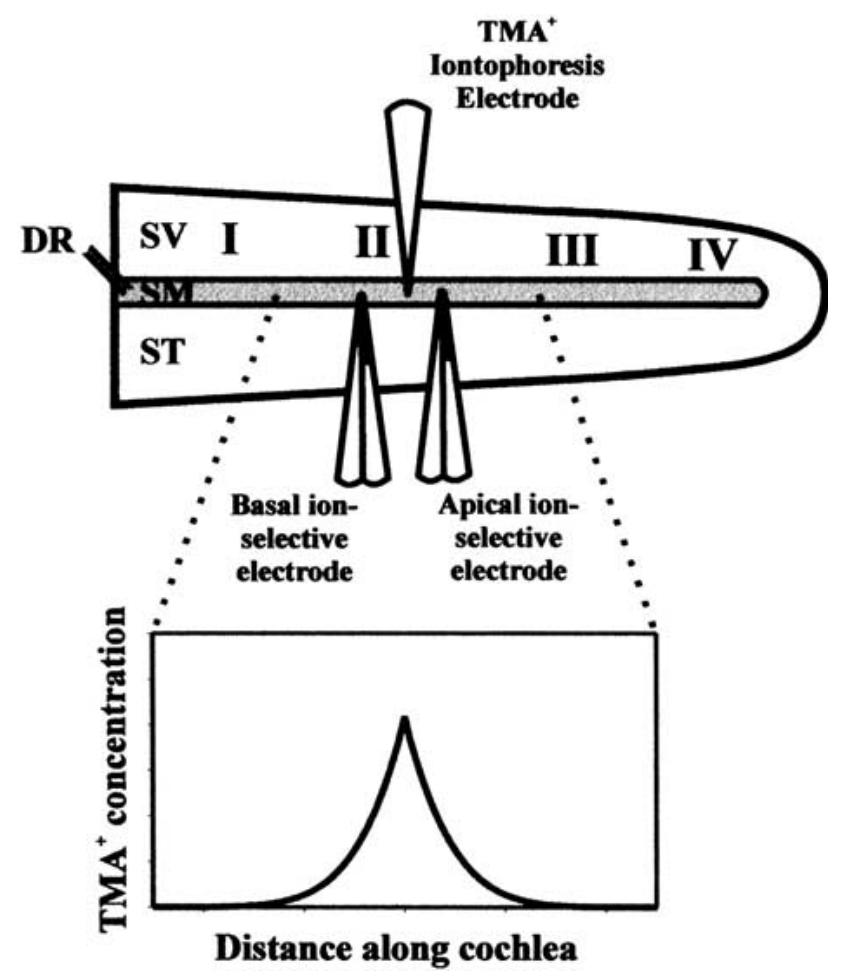

FIG. 1. Schematic of the location of electrodes in the cochlea, shown here uncoiled with the four turns marked with Roman numerals. The three scalae, scala tympani (ST), scala media (SM), and scala vestibuli (SV), and the ductus reunions (DR) are indicated. $\mathrm{TMA}^{+}$is injected into scala media by iontophoresis and sets up a concentration profile, as shown in the lower panel, as $\mathrm{TMA}^{+}$diffuses symmetrically away from the injection site. Two ion-selective electrodes are positioned in scala media approximately $0.5 \mathrm{~mm}$ basal and $0.5 \mathrm{~mm}$ apical to the injection site. Based on the concentration profile shown, longitudinal endolymph movements would cause oppositely directed concentration changes at the two electrodes while cross-sectional area changes would cause similar changes at both electrodes.

Analysis of marker changes used techniques that are described in detail elsewhere (Salt and DeMott 1995) and are presented briefly here. During the iontophoretic injection of volume marker, a concentration profile of the marker along the endolymphatic space is established, as shown schematically in Figure 1. Concentration is highest at the injection site and decreases with distance in both directions along the scala from the injection site as the marker spreads by diffusion. With this profile established, any longitudinal movement of endolymph will cause concentration at one ion-selective electrode to increase while the other decreases. For example, a basal movement of endolymph will cause concentration at the basal electrode to increase while the concentration at the apical electrode decreases. Thus, the ratio of concentrations detected by the two electrodes is a sensitive indicator of longitudinal endolymph flow. On the other hand, a cross-sec- 
tional area change, with fluid movement into or out of the compartment while the total amount of marker remains unchanged, will cause similar concentration changes at both electrodes. If fluid volume is lost and cross-sectional area decreases, then the marker concentration will increase. These relationships permit effects of flow and cross-sectional area change to be differentiated. Analysis of the marker concentration changes in those experiments using two ion-selective electrodes utilized our finiteelement model of the cochlear fluid spaces that combines the effects of diffusion, longitudinal volume flow, and clearance of marker (Salt and DeMott 1995). A version of this program is available on the Internet at oto.wustl.edu/cochlea/. A custom version of this program (not available on the Internet) was used to simulate the spread of marker along the endolymphatic space for the two electrode locations specific to each experiment. The diffusion coefficients used were $1.34 \times 10^{-9} \mathrm{~m}^{2} / \mathrm{s}$ for $\mathrm{TMA}^{+}$and 1.30 $\times 10^{-9} \mathrm{~m}^{2} / \mathrm{s}$ for $\mathrm{AsF}_{6}{ }^{-}$. For $\mathrm{TMA}^{+}$a clearance halftime of $30 \mathrm{~min}$ was used (Salt and DeMott 1995). After each $10 \mathrm{~s}$ period of simulation, the program compared the concentrations at the locations in the model corresponding to the recording locations, with the concentrations measured experimentally. First, the ratio of the concentrations between the two sites in the model was adjusted by longitudinal movements of marker, with an algorithm simulating longitudinal endolymph flow. If the basal/apical ratio in the model was lower than that observed experimentally, then a basal movement of marker was performed as required to obtain the exact ratio measured. The amount of longitudinal movement (i.e., longitudinal flow) necessary to keep the ratio of concentrations in the model identical to the measured ratio of concentrations was established. Then, the cross-sectional area of the compartment was adjusted in the model until the concentrations at the two locations in the model were identical to those for the experimental data. If the concentration in the model was too high, then cross-sectional area was decreased proportionately. In this manner, the simulation established a pair of concentration time courses identical to the measured data and provided sequential values for the longitudinal movements and cross-sectional area changes required to fit the measured data. Between each adjustment interval, corresponding to the $10 \mathrm{~s}$ data sampling intervals, the program simulated marker injection by iontophoresis and marker diffusion. In the case of $\mathrm{TMA}^{+}$ simulations, $\mathrm{TMA}^{+}$clearance from the endolymphatic space was also included. This analysis makes the assumption that flow and area changes are similar at the two recording electrode locations. Since the two sites are only approximately $1 \mathrm{~mm}$ apart (the distances are highly exaggerated in Fig. 1), this assumption appears reasonable.

The Animal Studies Committee of Washington University approved the experimental procedures used in this study under protocol numbers 91062 and 19990029.

\section{RESULTS}

An example experiment in which marker recordings were made during low-frequency stimulation of the ear is shown in Figure 2. Starting at zero time, $\mathrm{TMA}^{+}$ was iontophoresed into the endolymphatic space of the second turn while two $\mathrm{TMA}^{+}$-selective electrodes simultaneously monitored $\mathrm{TMA}^{+}$-concentration a small distance basally or apically relative to the injection site. In the experiment shown, the distances between the iontophoretic injection site and the recording sites were $0.4 \mathrm{~mm}$ and $0.375 \mathrm{~mm}$ for the basal and apical electrodes, respectively. The similar rate of concentration increase detected by the two electrodes is consistent with the near-symmetric placement of recording electrodes and the absence of appreciable longitudinal endolymph flow in the baseline state. The horizontal bars indicate times when $200 \mathrm{~Hz}$ tones were presented at the levels indicated for periods of $3 \mathrm{~min}$. At the 95 and $105 \mathrm{~dB}$ levels substantial EP changes were induced, but marker concentration changes were small. When the tone was presented at $115 \mathrm{~dB}$, however, substantial changes in marker concentration and an increase of EP during the exposure were observed. The concentrations at both recording sites decreased during the exposure and recovered back towards the pre-exposure level following exposure. Delivering the tone at $125 \mathrm{~dB}$ produced similar, but larger, changes.

Quantitative interpretation of the marker change, such as those in Figure 2, was provided by analysis of the data with a finite-element model as described in the Methods section. The cross-sectional area changes and longitudinal movements producing marker changes identical to those recorded are shown in Figure 3. With $115 \mathrm{~dB}$ stimulation the marker changes can be accounted for by a transient increase in cross-sectional area and a simultaneous basally directed displacement of endolymph. The indicated longitudinal displacement, being based on a change in the ratio of concentrations at the two recording locations, is a robust measure and provides strong support for an endolymph volume disturbance taking place.

The endolymph cross-sectional area changes and longitudinal movements produced by $115 \mathrm{~dB}$ SPL tones at 50, 200, 500, and $1000 \mathrm{~Hz}$ are summarized in Figure 4 . The most consistent findings were obtained 


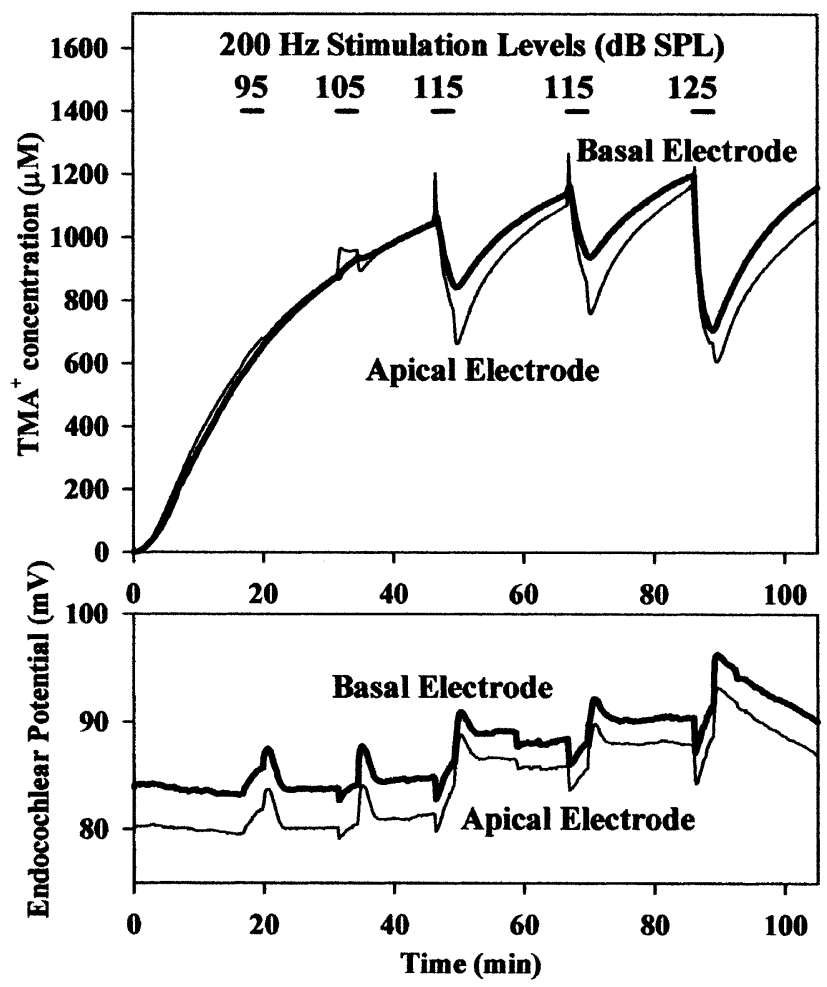

FIG. 2. Example experiment in which $\mathrm{TMA}^{+}$marker concentration changes were recorded simultaneously from two sites during exposure to $200 \mathrm{~Hz}$ tones at different levels. $\mathrm{TMA}^{+}$was injected iontophoretically throughout the experiment starting at zero time, producing a concentration increase that saturates with time. The simultaneously recorded EP measurements are shown in the lower panel. With an exposure level of $115 \mathrm{~dB}$ SPL, substantial decreases of marker are produced that are most pronounced at the apical electrode, and EP increases are generated. Stimulation with $125 \mathrm{~dB}$ SPL causes even larger $\mathrm{TMA}^{+}$and EP changes.

with $200 \mathrm{~Hz}$ stimulation. During the 3 min exposure to $200 \mathrm{~Hz}$, endolymph cross-sectional area increased, reaching a maximum of $33.7 \%$ (SD 20.5, $n=10$ ) immediately after the exposure. The recovery followed near-exponential kinetics with a single exponential curve fitted to the $200 \mathrm{~Hz}$ data indicating a recovery half-time of $3.2 \mathrm{~min}$. Simultaneous with the enlargement of the endolymphatic space was a basally directed displacement of endolymph that averaged approximately $0.3 \mathrm{~mm}$. Since there is approximately $8 \mathrm{~mm}$ of the endolymphatic space apical to the second turn, this would represent about $4 \%$ of the volume moving as flow. After the exposure, although the rate decreased, the displacement showed no indication of a reversal as endolymph cross section decreased. The EP showed a negative deflection during the exposure, which then moved positive and became greater than the pre-exposure level for a few minutes after exposure. At the higher frequencies tested (500 and $1000 \mathrm{~Hz}$ ), results were more variable, with some animals showing area changes comparable to those at $200 \mathrm{~Hz}$, while others showed almost no change.

\section{Cross-sectional area of the endolymphatic space}

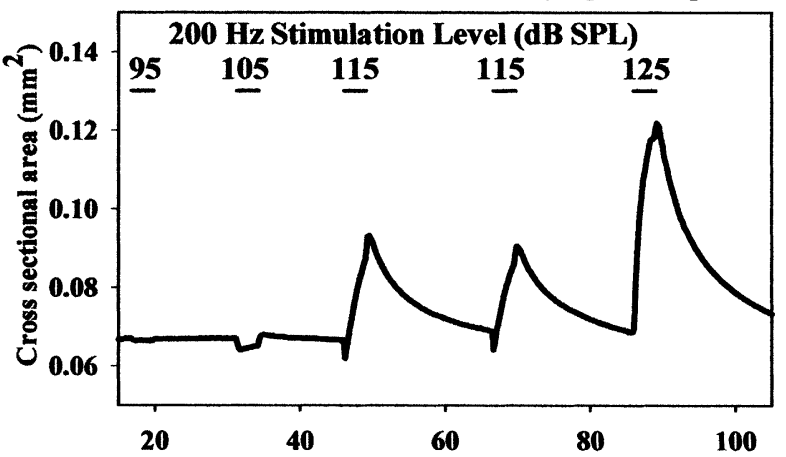

Longitudinal displacement of endolymph

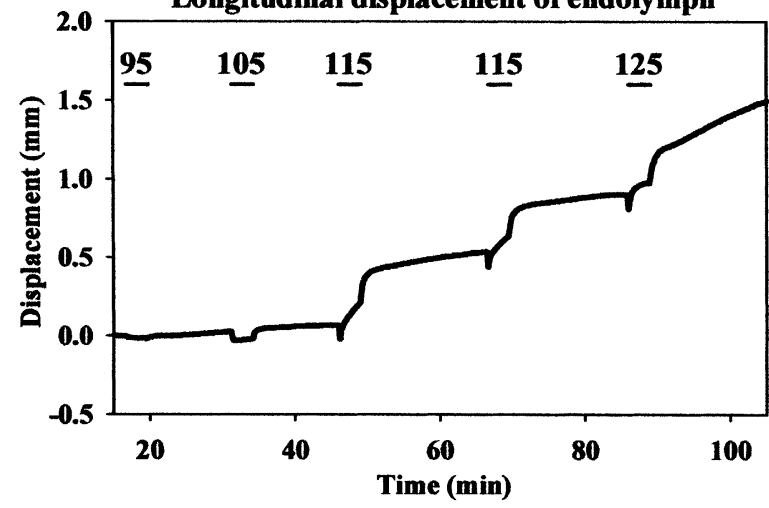

FIG. 3. Cross-sectional area and longitudinal movements of endolymph derived from the data in Figure 2 by simulating the experiment with a finite-element model of the endolymphatic space. Stimulation at levels of $115 \mathrm{~dB}$ and higher produce substantial area changes and basally directed displacements of endolymph in the second turn.

Longitudinal movements were also smaller. EP changes were larger but showed less tendency to show supernormality after exposure. With lower frequency of stimulation $(50 \mathrm{~Hz})$, area changes were consistent but smaller than those at $200 \mathrm{~Hz}$. Longitudinal movements were small and were apically directed, which is the opposite to that seen with $200 \mathrm{~Hz}$.

Since the 3-electrode experiment (1 injection electrode and 2 ion-selective electrodes) was technically difficult, we performed some parametric studies with only 1 ion-selective electrode on the apical side of the injection, the site where the greatest magnitude of marker change was observed. The dependence of the magnitude of sound-induced marker changes on stimulus level and frequency is summarized in Figure 5. For $200 \mathrm{~Hz}$ stimulation given at different levels, it is apparent that no marker changes were detectable with stimulation levels of $105 \mathrm{~dB}$ and lower. As sound level increased, the magnitude of the marker change increased. In a limited number of experiments, marker injections and recordings were made in the basal cochlear turn. Sound-induced marker changes in the basal turn were far smaller than those observed in the second turn, suggesting 


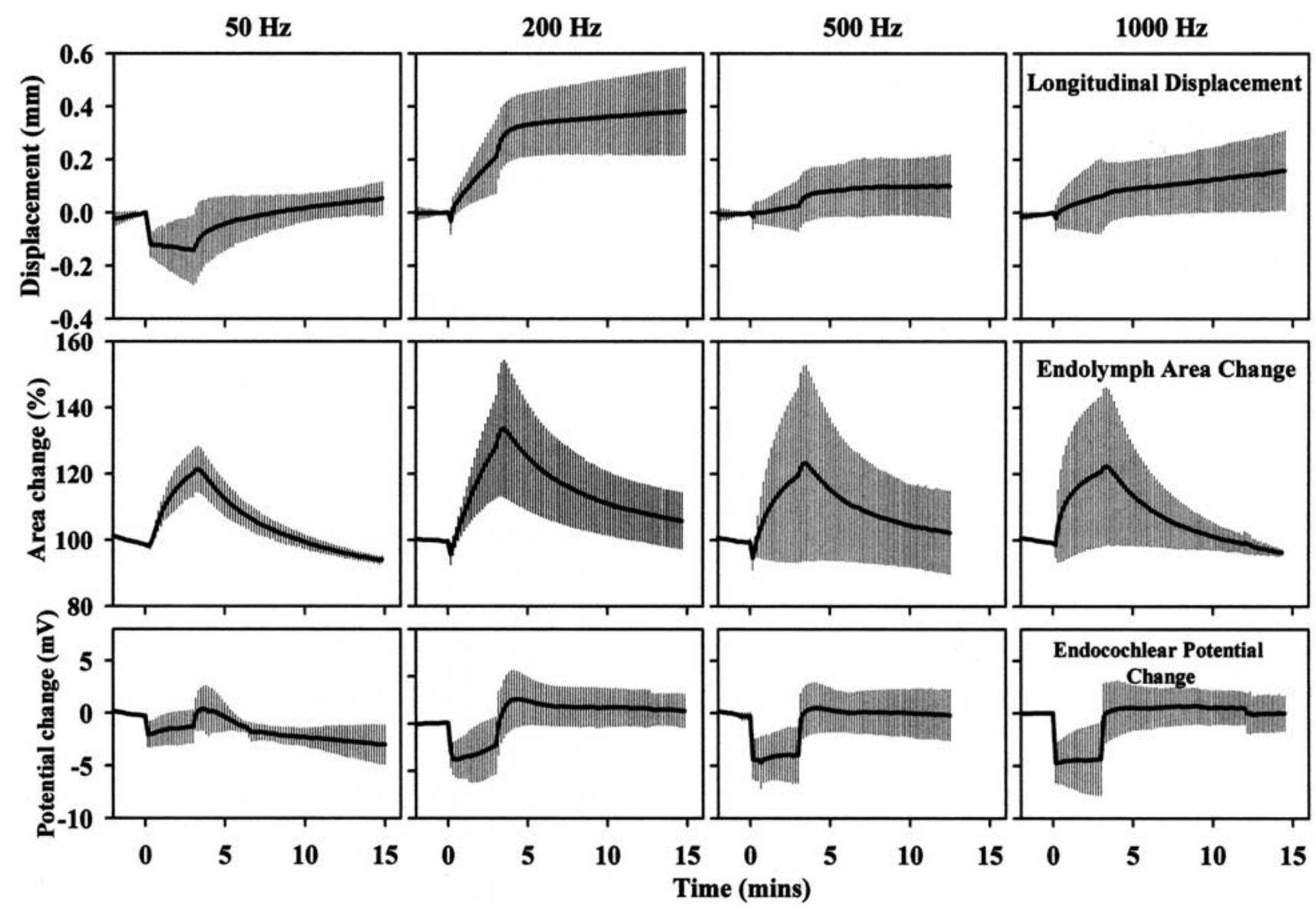

FIG. 4. Summary of the longitudinal displacements (upper row), cross-sectional area changes (middle row), and endocochlear potential changes (lower row) produced by 3 min stimulation with a $115 \mathrm{~dB}$ tone at the four frequencies indicated. Bars indicate standard deviation. The number of observations in each group were $50 \mathrm{~Hz}: 3$; $200 \mathrm{~Hz}$ : 10; $500 \mathrm{~Hz}$ : 4; $1000 \mathrm{~Hz}: 3$. The EP data for each experiment was taken as the average of the two traces recorded at the basal and apical electrode locations.

volume recovers back toward normal. The experiment also provides a technical control, as the $\mathrm{K}^{+}$ measurements were made with ion-selective electrodes virtually identical to those for $\mathrm{TMA}^{+}$, so that the possibility of some nonspecific effect of the sound causing substantial potential changes of the ion electrode is excluded.

While the flow measurements are robust, there are a number of processes other than cross-sectional area change that could potentially give rise to marker concentration decreases. A concern arose whether the marker decreases we observed in fact represented cross-sectional area increases of the endolymphatic space or whether they could be explained by other factors. One possibility explored was that the vibration caused by $200 \mathrm{~Hz}$ could possibly "stir" the fluid in the endolymphatic compartment, so that the marker decrease near the injection site could represent an increased rate of dispersal along the scala. A simulation of this process is illustrated in Figure 7, where the left panel shows the calculated concentration time courses resulting from a 3 min period in 


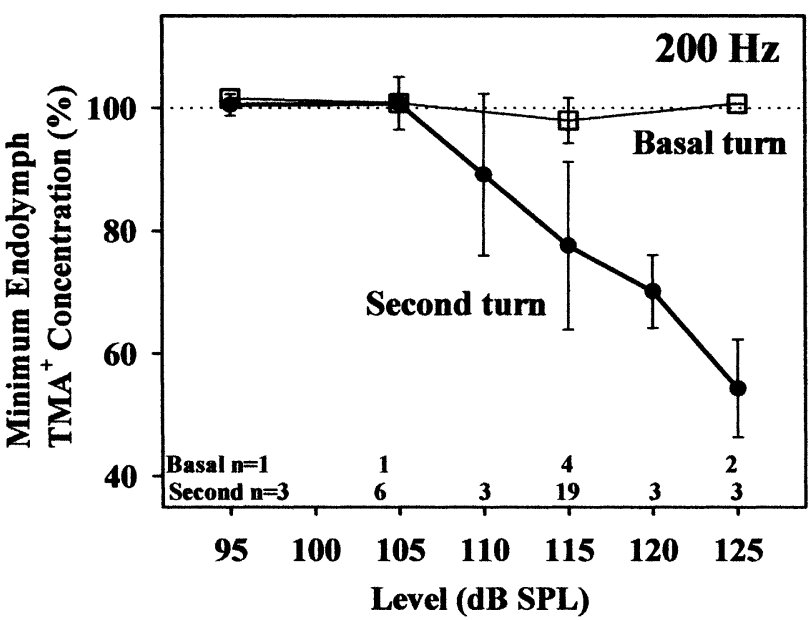

FIG. 5. Summary of the magnitude of marker concentration changes seen with exposure to a $200 \mathrm{~Hz}$ stimulation for $3 \mathrm{~min}$ at varying levels (left panel) and to tones of varying frequency for $3 \mathrm{~min}$ at $115 \mathrm{~dB}$ SPL (right panel). The concentration present prior to exposure is defined as

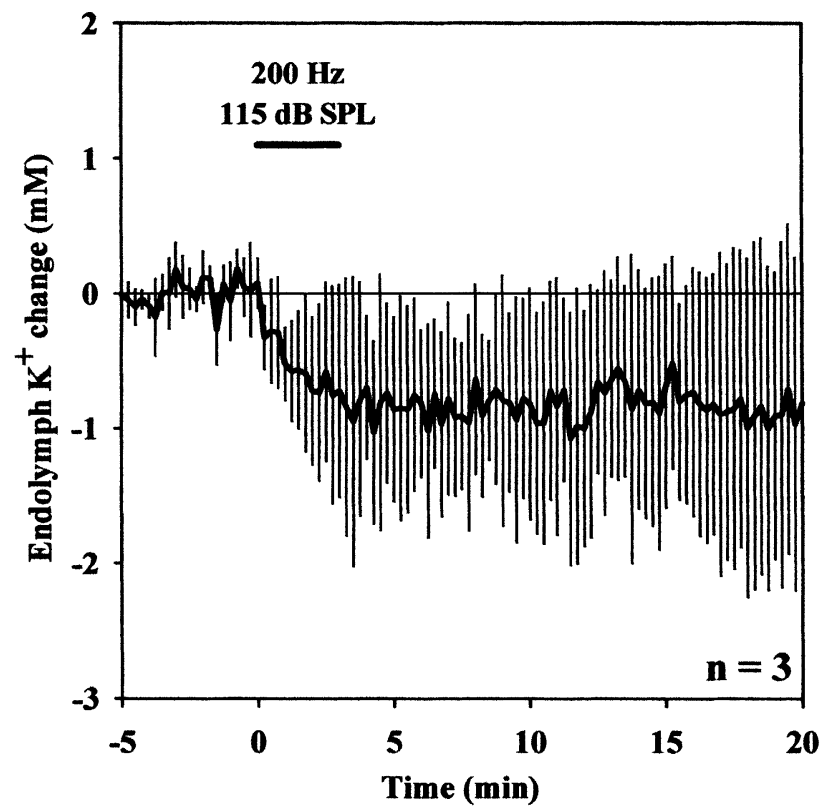

FIG. 6. Changes of endolymph $\mathrm{K}^{+}$recorded in three experiments during exposure to $200 \mathrm{~Hz}$ tones at $115 \mathrm{~dB}$ SPL for $3 \mathrm{~min}$ as indicated. The mean endolymph $\mathrm{K}^{+}$level prior to stimulation was 151.2 $\mathrm{mM}$ (SD 10.3 mM). Bars indicate standard deviation of the normalized change.

which the diffusion coefficient was increased by a factor of 10 . The simulation shows the expected decrease in concentration near the injection site, but it also shows that for recording distances further away, the concentration should increase as the marker spreads to that region more quickly. The right panel of Figure 7 shows an experiment in which $\mathrm{TMA}^{+}$was recorded simultaneously in the basal and second turns during marker injection into the second turn

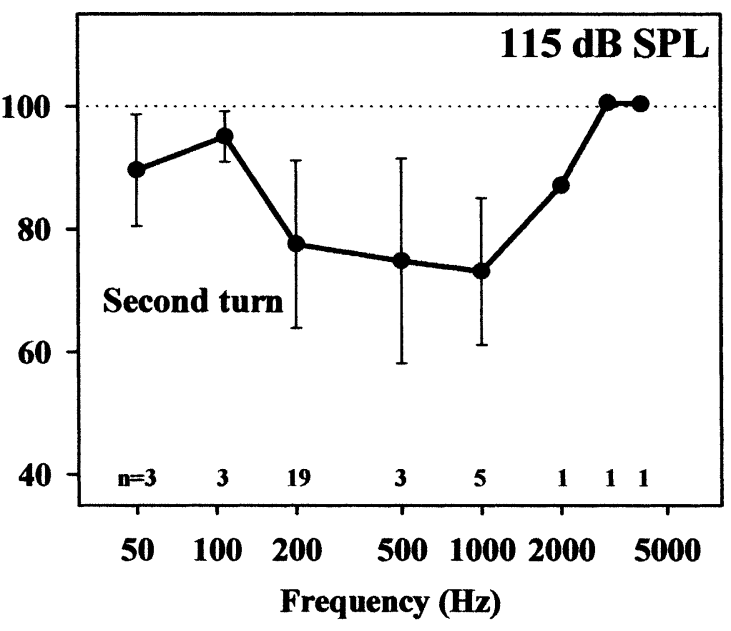

100 and the value shown is the minimum $\mathrm{TMA}^{+}$concentration in the period during and following the tone. The recording electrodes were all positioned apical to the $\mathrm{TMA}^{+}$injection site. Bars indicate standard deviation of the number of observations indicated.

and a $200 \mathrm{~Hz}$ tone exposure. A sound-induced reduction in marker concentration was observed in the second turn but no change in rate of marker increase was observed in the basal turn, even though the measured marker level was increasing slowly at the time.

A second possibility considered was that the rate of clearance of $\mathrm{TMA}^{+}$from endolymph could be increased by the sound exposure, causing the marker concentration to fall. This was thought unlikely, as the recovery of marker concentration after sound exposure would not be expected if marker was released from the compartment. Nevertheless, the possibility was tested by using a marker with completely different clearance characteristics. Since $\mathrm{TMA}^{+}$is a cation, its loss from the endolymphatic space is driven by the EP. In contrast, the marker $\mathrm{AsF}_{6}{ }^{-}$is an anion and is retained in the endolymphatic space by the EP. In simulations of $\mathrm{AsF}_{6}{ }^{-}$dispersal in endolymph, virtually no clearance is necessary to account for the dispersal time course (Salt et al. 1995). Figure 8 shows the results from an experiment in which $\mathrm{AsF}_{6}{ }^{-}$was used as the marker from which flow and area changes were derived. Virtually the same time courses were obtained for both displacement and area change, even though no clearance of $\mathrm{AsF}_{6}{ }^{-}$was incorporated into the analysis. These findings exclude the possibility that the marker concentration decreases result from sound-induced permeability changes of the endolymphatic boundary.

In their initial report, Kirk and Patuzzi (1997) monitored AP thresholds at $4 \mathrm{kHz}$ and emissions with primaries at 3.4 and $4 \mathrm{kHz}$ or 12 and $14 \mathrm{kHz}$ during $200 \mathrm{~Hz}$ exposures. They noted that AP threshold improvements and emissions changes occurred with $200 \mathrm{~Hz}$ exposure levels as low as $85 \mathrm{~dB}$ SPL. In our 

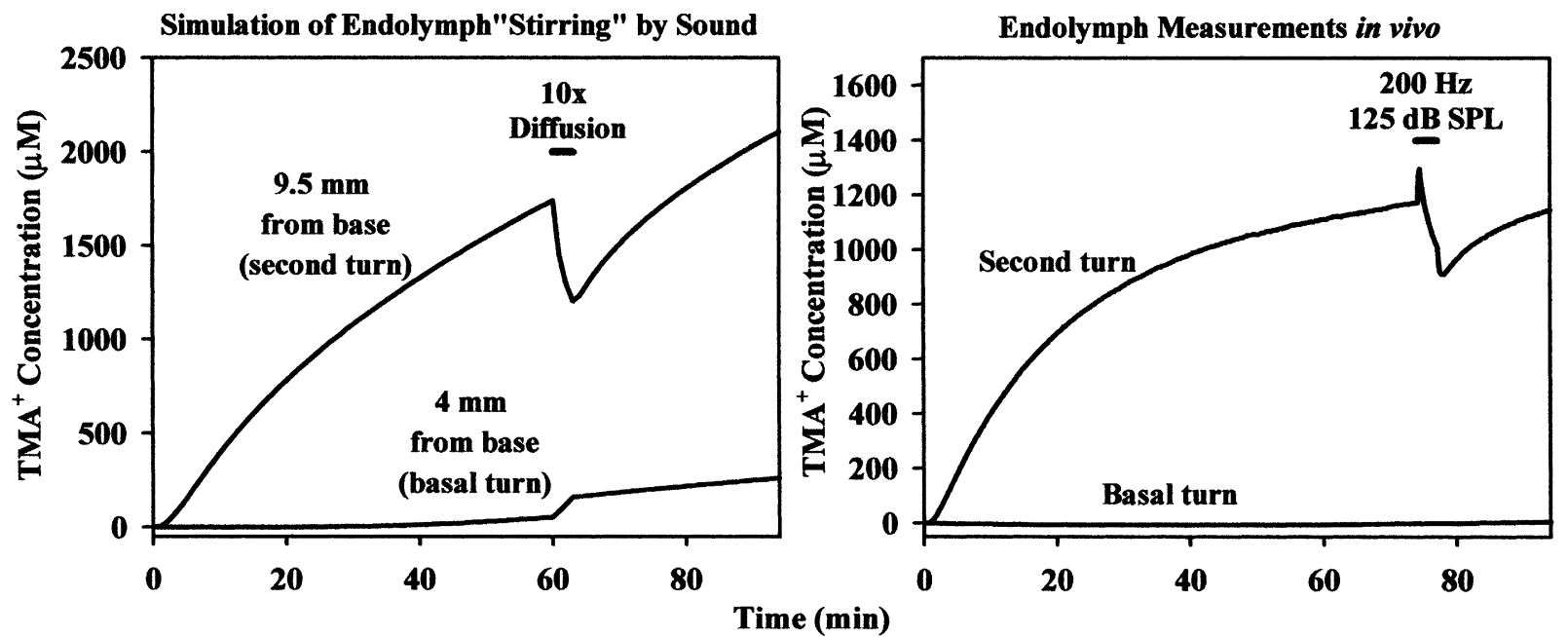

FIG. 7. Control experiment to test for the possibility that the $200 \mathrm{~Hz}$ stimulation caused a "stirring" of scala tympani. Left Prediction from the finite-element model of cochlear fluids in which a second-turn injection (10 mm from base) is simulated with concentration recordings made $0.5 \mathrm{~mm}$ from the injection site $(9.5 \mathrm{~mm}$ from base) and $6 \mathrm{~mm}$ from the injection site $(4 \mathrm{~mm}$ from base, corresponding to a basal turn recording). When the diffusion coefficient was increased

by a factor of 10 for $3 \mathrm{~min}$, the marker decreases at the site close to the injection and increases at the distant site. Right A comparable experiment performed in vivo, with simultaneous recordings in the basal and second turn during $200 \mathrm{~Hz}$ stimulation. Although the marker decreases in the second turn, no increase in the basal turn is observed, showing that the marker decreases do not occur as a result of endolymph "stirring."
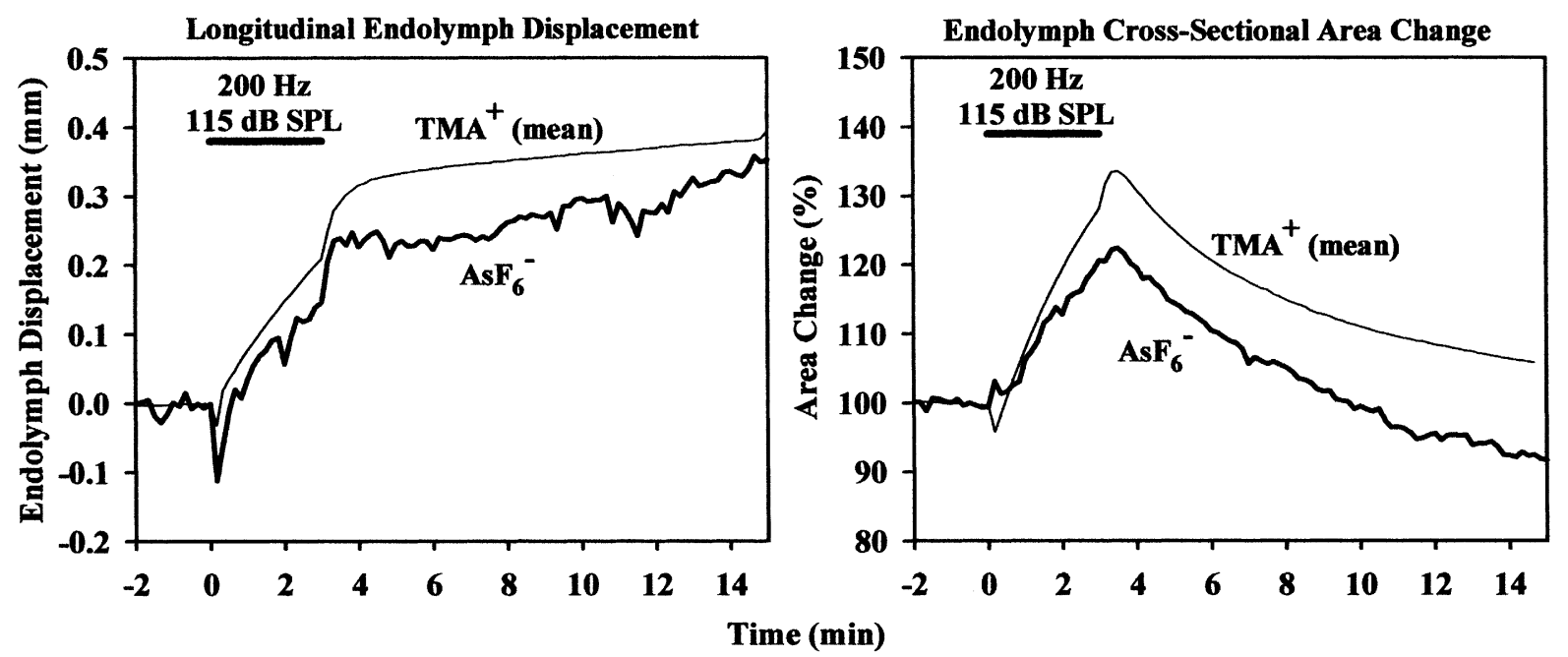

FIG. 8. Control to test for the possibility of sound-induced changes in marker clearance contributing to marker concentration changes. Endolymph displacement (left) and cross-sectional area changes (right) derived from an experiment in which the anionic marker $\mathrm{AsF}_{6}{ }^{-}$was

used are shown to be comparable to those from experiments using $\mathrm{TMA}^{+}$. $\mathrm{TMA}^{+}$and $\mathrm{AsF}_{6}{ }^{-}$have completely different clearance characteristics. $\mathrm{TMA}^{+}$is cleared with a half-time of $30 \mathrm{~min}$, which is incorporated into the analysis, and $\mathrm{AsF}_{6}{ }^{-}$shows no clearance at all.

experiments, we found evidence of endolymph volume changes only with $200 \mathrm{~Hz}$ exposures at $110 \mathrm{~dB}$ and higher. This raises the possibility that endolymph volume changes may not be the cause of the threshold and emissions changes. To ensure there was no systematic difference between our two studies, such as a calibration difference, we therefore examined the sensitivity of AP thresholds to $200 \mathrm{~Hz}$ exposures. Figure 9 shows the time courses of AP threshold changes measured at three probe frequencies in response to $200 \mathrm{~Hz}$ exposures at $115 \mathrm{~dB}$ SPL (upper

panel) and $95 \mathrm{~dB}$ SPL (lower panel). The average pre-exposure thresholds were $29.8 \mathrm{~dB}$ SPL (SD 6.5, $n=50$ ), $31.6 \mathrm{~dB}$ SPL (SD 3.5, $n=27$ ), and $46.0 \mathrm{~dB}$ SPL (SD $4.2, n=23$ ) at 8,4 , and $2 \mathrm{kHz}$, respectively. For comparison, the EP changes induced by the two levels of $200 \mathrm{~Hz}$ stimulation are shown. The EP changes were not recorded from the same group of animals as the AP thresholds, but were from those in which marker measurements were made. We found threshold fluctuations following the exposure, with the greatest threshold improvement occurring 2- 

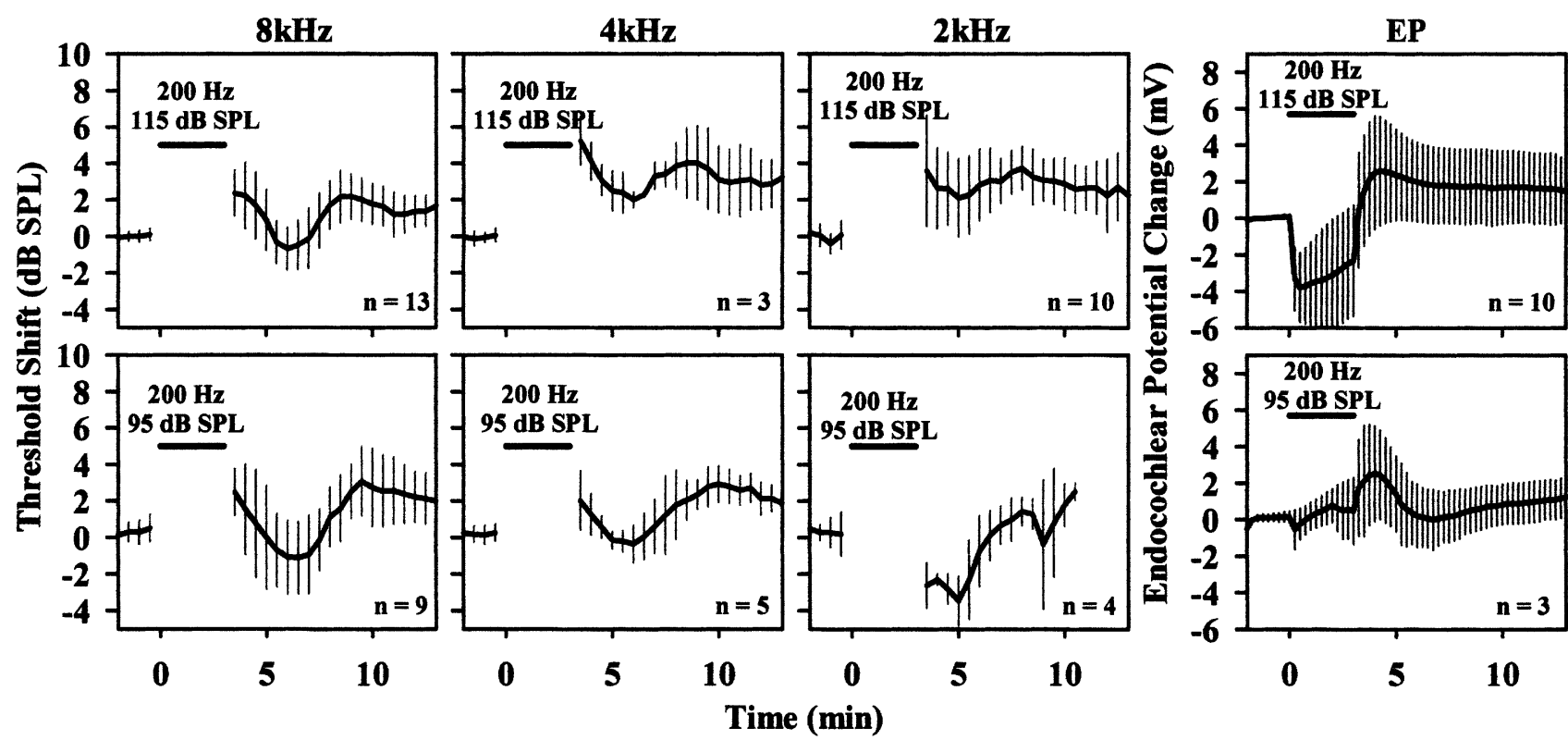

FIG. 9. Threshold shifts at 8,4 , and $2 \mathrm{kHz}$ and EP changes resulting from $200 \mathrm{~Hz}$ tone exposures for 3 min at $115 \mathrm{~dB}$ SPL (upper row) and 95 dB SPL (lower row). Bars indicate standard deviation. EP and threshold changes were recorded in different experimental groups.

3 min postexposure. These findings are comparable to those of Kirk and Patuzzi (1997). Threshold fluctuations occurred with a $95 \mathrm{~dB}$ SPL stimulus level, a level that did not cause detectable volume changes. With higher stimulus levels that were shown to cause endolymphatic hydrops, the elevation of AP thresholds was small. The time course of AP threshold changes appears similar for $95 \mathrm{~dB}$ and $115 \mathrm{~dB}$ stimulus levels and is quite different from the time course of endolymph cross-section change seen in Figure 4. A supernormality of EP was observed after exposure at both levels although the time course of EP change appeared to be faster than that of the AP threshold change.

The sensitivity of AP threshold shifts to the level of the $200 \mathrm{~Hz}$ tone is summarized in Figure 10. Postexposure threshold improvements were found for a broad range of exposure levels, which is comparable to the observations of Kirk and Patuzzi (1997). Only when the applied tone level was $125 \mathrm{~dB}$ was there marked threshold elevation, which is presumed to represent a temporary threshold shift (TTS) as generally associated with loud sound exposure.

\section{DISCUSSION}

Our results show that low-frequency tones, presented at high but nondamaging levels, cause an acute endolymphatic hydrops in the second turn of the guinea pig cochlea. This confirms the observation by Flock and Flock (2000) of low-frequency sound-
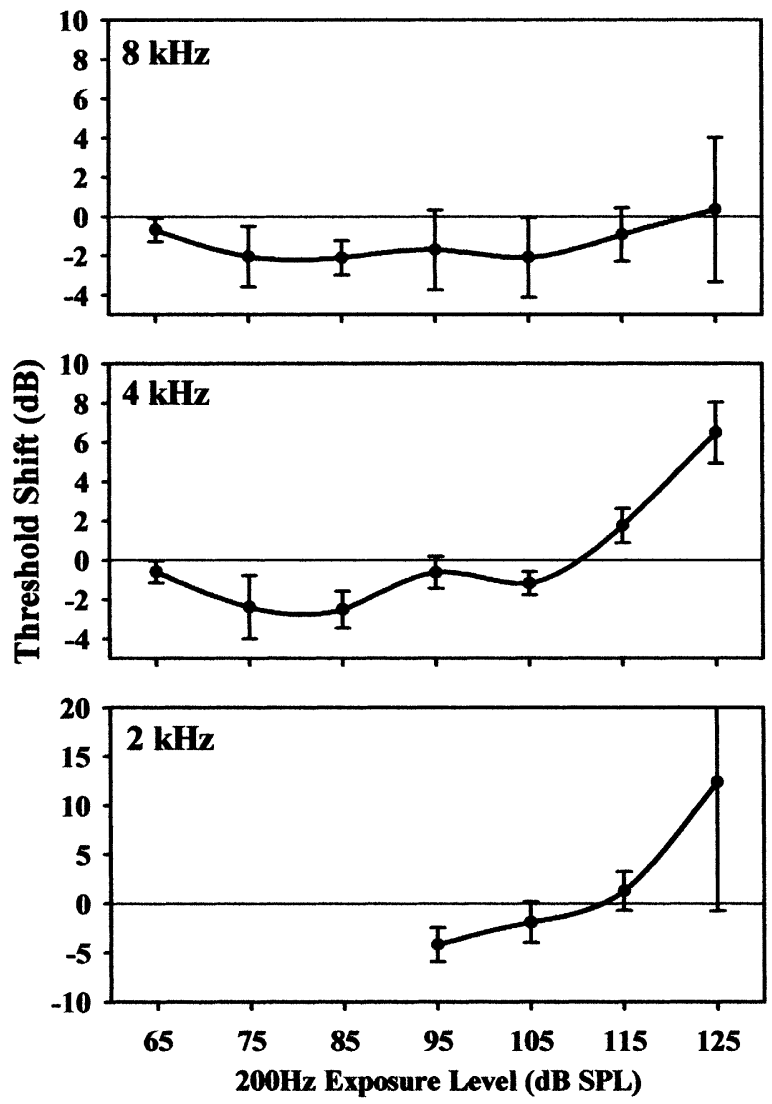

FIG. 10. Summary of the minimum AP thresholds obtained in the period immediately following $200 \mathrm{~Hz}$ exposures at levels from 65 to $125 \mathrm{~dB}$ SPL. Thresholds were measured at 8, 4, and $2 \mathrm{kHz}$. Bars indicate standard deviation. 
induced hydrops in the apical turn of isolated, perfused cochleae. The further observation of near-normal AP thresholds at a time when endolymphatic hydrops is present in the cochlea suggests that this type of hydrops is not a pathologic state, but is part of the normal repertoire of cochlear responses to stimulation. Recovery from the hydropic state takes place rapidly, apparently dominated by local homeostatic mechanisms. Although a small basally directed flow was observed during the 3 min exposure period, the flow rate is small relative to the area increase and the rate falls after exposure. The recovery back toward normal cross-sectional area therefore cannot be accounted for by longitudinal flow mechanisms. The basally directed flow during $200 \mathrm{~Hz}$ exposure is consistent with prior studies in which basally directed flow was demonstrated following acute endolymphatic injections (Salt and DeMott 1997) and from pressure studies that suggest that the saccule may form a compliant structure so that flow may be directed toward it (Wit et al. 2000). However, such an explanation based on "passive" mechanical properties of the endolymphatic space is not consistent with coexistence of apically directed displacement and endolymphatic hydrops induced by $50 \mathrm{~Hz}$ stimulation. This suggests that more complex relationships, possibly involving compliance changes of the boundary tissues, may exist. Indeed, the observation that marker changes were minimal in the basal turn also suggests that the hydrops induced in the second turns [and, based on Flock and Flock (2000), also assumed to be present in higher turns] does not spread down the cochlea to the saccule as endolymph injection experiments have suggested (Salt and DeMott 1997, Wit et al. 2000). This may be the result of the nonlinear relationship between endolymph pressure and volume increase (Wit et al. 2000), in which the endolymphatic boundaries appear to be highly compliant for small volume disturbances.

The magnitude and rate of endolymph volume change induced by $200 \mathrm{~Hz}$ exposure is larger than we thought possible, based on steady-state kinetic data. The observation that endolymph volume can increase by more than $30 \%$ within $3 \mathrm{~min}$, with almost no reduction in endolymph $\mathrm{K}^{+}$concentration (approximately $0.6 \%$ decrease), indicates that electrolytes must be added to the endolymphatic space during the exposure and subsequently removed during the recovery period. The rate of these changes (half-time $2.5 \mathrm{~min}$ for the increase, $3.2 \mathrm{~min}$ for the recovery; based on fitted exponential curves) is much faster than the endolymph turnover rates measured by radiotracer under steady-state conditions. In guinea pigs the turnover half-time for endolymph $\mathrm{K}^{+}$was $55 \mathrm{~min}$ and for $\mathrm{Cl}^{-}$it was $69 \mathrm{~min}$ (Konishi et al. 1978; Konishi and Hamrick 1978). The rate of ionic change with low-frequency stimulation is thus larger than that expected based on steady-state endolymph turnover rates.

The volume changes we observed must arise from an accumulation of both anions and cations within the endolymphatic space, as we see only minor changes in endolymph $\mathrm{K}^{+}$concentration during $200 \mathrm{~Hz}$ exposures at $115 \mathrm{~dB}$ SPL. Perilymphatic manipulations with osmotically altered media indicate that water equilibration is rapid between the endolymphatic and the perilymphatic spaces (Salt and DeMott 1995). The equilibration half-time was estimated to be approximately $8 \mathrm{~min}$, so that an increase in total electrolytes in the endolymph would result in a volume increase. In order for electrolytes to accumulate there must be a reduction in $\mathrm{K}^{+}$efflux from the endolymphatic space during the tone exposure. Based on measurements of transducer operating point during low-frequency simulation, Kirk and Patuzzi (1997) suggested that there is a movement of the organ of Corti toward scala tympani as a result of high level, low-frequency tone stimulation. Such a movement would reduce current through the organ of Corti, a current that is predominantly carried by $\mathrm{K}^{+}$ as the hair cell transducer channels are cation-specific (Kros 1996). The second factor is how the increase of endolymph anion content could arise. Zidanic and Brownell (1992) demonstrated that, during individual stimulus cycles as current through the organ of Corti was modulated, current efflux through Reissner's membrane was modulated in a reciprocal manner. Current through Reissner's membrane was shown to increase when current through the organ of Corti was reduced. They interpreted this as indicating that the stria vascularis maintained a constant current during acoustic stimulation and that Reissner's membrane acted as a current shunt, compensating for current changes through the organ of Corti. They also noted that, in the absence of stimulation, voltage gradients in the fluids of scala vestibuli were larger than those in scala tympani (Zidanic and Brownell 1990), indicating higher current magnitudes in scala vestibuli. While part of this apparently sizable current through Reissner's membrane may be carried by $\mathrm{K}^{+}$, there is considerable evidence that $\mathrm{Cl}^{-}$movements across Reissner's membrane also occur. In a series of studies, evidence was found for the presence of cAMP-activated $\mathrm{Cl}^{-}$channels in Reissner's membrane that, when activated by forskolin, produced increases in endolymph $\mathrm{Cl}^{-}$concentration (Doi et al. 1992; Kitano et al. 1993, 1995). Chloride channels have also been demonstrated in the apical membranes of Reissner's membrane epithelial cells (Yeh et al. 1997) If a substantial part of the outwardly directed current observed by Zidanic and Brownell (1990) was carried by $\mathrm{Cl}^{-}$entering the endolymphatic space, then the 
situation could arise in which a reduction in $\mathrm{K}^{+}$current across the organ of Corti would result in an increase in $\mathrm{Cl}^{-}$current across Reissner's membrane. This would result in both $\mathrm{K}^{+}$and $\mathrm{Cl}^{-}$accumulating in the endolymphatic space. There could also be a degree of positive feedback in this process since volume enlargement would displace the organ of Corti even further toward scala tympani. Although the mechanisms of endolymph $\mathrm{Cl}^{-}$homeostasis are not well established, it is notable that the radiotracer studies cited above show that the rate of endolymph $\mathrm{Cl}^{-}$ turnover (69 min half-time) is almost as fast as that of $\mathrm{K}^{+}$(55 min half-time), so the contribution of $\mathrm{Cl}^{-}$ movements to the currents circulating through endolymph must be considerable even in this undisturbed state. However, the above explanation is obviously speculative to some degree and should not be regarded either as complete or as the sole explanation. The possible contributions of other tissues and cell types and other cations, such as $\mathrm{HCO}_{3}{ }^{-}$, cannot be excluded. Other plausible mechanisms involved in the volume increase may also exist.

The nature of the operating point shift occurring with low-frequency tones is of considerable interest. Kirk and Patuzzi (1997) suggested that either "an osmotic imbalance in the fluids of the organ of Corti" or a "bloating of scala media" could be the cause of the operating point shift. While the acute endolymphatic hydrops we observe could certainly contribute to an operating point shift (tending to displace the organ of Corti toward scala tympani and reducing current through the organ of Corti), it appears that operating point shifts occur at stimulus levels well below those causing endolymph volume disturbances. It remains likely that the operating point shift at the level of the hair cells is a major factor in the cause of the endolymph volume disturbance, but it appears less likely that the operating point shift occurs as a result of endolymph volume disturbance. It is not possible to draw a strong conclusion regarding cause and effect from the present data as the organ of Corti may be more sensitive to small volume disturbances than is our marker measurement system. Localized ionic changes in the organ of Corti may precede disturbances of endolymph volume. This may also account for why threshold changes to high-frequency stimulation $(8 \mathrm{kHz})$ are observed under conditions where endolymph volume in the basal turn is apparently unaffected.

The possible effect of EP changes on the cochlear sensitivity and on endolymph volume changes remains uncertain. EP changes are known to strongly influence auditory sensitivity, with thresholds elevated by approximately $1 \mathrm{~dB}$ for each $1 \mathrm{mV}$ of EP decrease (Sewell 1984). It is therefore possible that the poststimulus EP increase could contribute to thresh- old improvements after sound. However, the time course of AP threshold changes is slower than that of the EP (Fig. 9). In addition, Kirk and Patuzzi (1997) pointed out that EP changes would also be a direct consequence of operating point shifts of the organ of Corti. A displacement of the organ of Corti toward scala tympani would reduce current through the hair cells and cause a rise in EP. Their analysis indicated that the sensitivity changes of cochlear responses were more consistent with operating point shifts at the level of the hair cells being the primary cause, with EP changes arising as a secondary phenomenon. Our present data remain consistent with this interpretation. Similarly, while substantial ionic movements must be associated with endolymph volume changes and their recovery, the EP changes are only a few $\mathrm{mV}$ in magnitude and follow time courses dissimilar to those of the volume changes. Since the potential changes cannot be accounted for simply by modulation of $\mathrm{K}^{+}$currents at the hair cells or stria vascularis, additional data will be required to understand how such large ionic movements can occur with only modest influence on the EP.

The physiologic effects of acute hydrops seen in the present study are considerably different from those associated with the chronic hydrops that develops over a period of weeks after ablation of the endolymphatic sac. Associated with the acute hydrops in the present study is an increase in the postexposure EP, while EP in the chronic hydrops model is typically decreased by $5-30 \mathrm{mV}$ (Cohen and Morizono 1984; Salt et al. 1995). The observation that the thresholds at 8,4 , and $2 \mathrm{kHz}$ are within $5 \mathrm{~dB}$ of normal in animals in which endolymph volume has increased by over $30 \%$ clearly demonstrates that hydrops itself is not the cause of substantial hearing loss. Threshold shifts in animals with chronic hydrops are generally greater and increase with time (reviewed by Horner 1993). However, a number of studies have reported normal thresholds or only slight threshold elevations in animals with confirmed endolymphatic hydrops (van Deelen et al. 1987; Klis and Smoorenburg 1988). It seems likely that the substantial hearing loss seen in the later stages of chronic hydrops involves factors other than the endolymph volume increase. This may include the EP decrease, which is known to elevate cochlear sensitivity (Sewell 1984), endolymph $\mathrm{Ca}^{2+}$ elevation (Ninoyu and Meyer zum Gottesberge 1986; Salt and DeMott 1994), or other pathological changes known to be occurring in the cochlea, such as stereociliary abnormalities (Horner et al. 1988). In addition, our observations showing that acute endolymphatic hydrops is rapidly corrected in the normal cochlea, apparently by local ion transport mechanisms, raises the intriguing question of why such a rapid correc- 
tion does not occur in the cochleae of animals after endolymphatic sac ablation. Understanding the mechanisms contributing to local volume recovery, specifically what factors block or stimulate recovery from volume disturbance, would seem to be of prime importance for understanding chronic hydrops and Ménière's disease.

\section{ACKNOWLEDGMENTS}

I would like to dedicate this article to the memory of Des Kirk and Graeme Yates, both outstanding physiologists who pioneered work in this area. Their contributions to the field will be missed. This study was supported by research grant RO1 DC01368 from the National Institute on Deafness and Other Communication Disorders (NIDCD), National Institutes of Health. The technical contributions of John DeMott and Shane Hale, who performed the experimental work reported here, are also acknowledged.

\section{REFERENCE}

Cohen J, Morizono T. Changes in EP and inner ear ion concentrations in experimental endolymphatic hydrops. Acta Otolaryngol. 98:398-402, 1984.

Doi K, Mori N, Matsunaga T. Adenylate cyclase modulation of ion permeability in the guinea pig cochlea: A possible mechanism for the formation of endolymphatic hydrops. Acta Otolaryngol. 112:667-673, 1992.

FLOCK A, FlOCK B. Hydrops in the cochlea can be induced by sound as well as by static pressure. Hear. Res. 150:175-188, 2000.

GREENWOOD DD. A cochlear frequency-position function for several species-29 years later. J. Acoust. Soc. Am. 87:2592-2605, 1990.

HiRsh IJ, WARD WD. Recovery of the auditory threshold after strong acoustic stimulation. J. Acoust. Soc. Am. 24:131-141, 1952.

Horner KC, Guilhaume A, Cazals Y. Atrophy of middle and short stereocilia on outer hair cells of guinea pig cochleas with experimentally induced hydrops. Hear. Res. 32:41-48, 1988.

KEMP DT. Otoacoustic emissions, travelling waves and cochlear mechanisms. Hear. Res 22:95-104, 1986.

KiRk DL, PAtuzzi RB. Transient changes in cochlear potentials and DPOAEs after low-frequency tones: the "two-minute bounce" revisited. Hear. Res. 112:49-68, 1997.

Kirk DL, Moleirinho A, Patuzzi RB. Microphonic and DPOAE measurements suggest a micromechanical mechanism for the "bounce" phenomenon following low-frequency tones. Hear. Res. 112:69-86, 1997.

Kitano I, Doi K, Mori N, Matsunaga T. Involvement of $\mathrm{Cl}^{-}$transport in forskolin-induced elevation of endocochlear potential. Hear. Res. 71:23-27, 1993.

Kitano I, Mori N, Matsunaga T. Role of endolymphatic anion transport in forskolin-induced $\mathrm{Cl}^{-}$activity increase in scala media. Hear. Res. 83:37-42, 1995.
KLIS JF, SMoorenburg GF. Cochlear potentials and their modulation by low frequency sound in early endolymphatic hydrops. Hear. Res. 32:175-184, 1988.

Kros CJ. Physiology of mammalian cochlear hair cells. In: Dallos P, Popper AN, Fay RR (eds) The Cochlea. Springer, New York, pp 318-385, 1996.

Konishi T, HAMrick PE. Ion transport in the cochlea of guinea pig. II. Chloride transport. Acta Otolaryngol. 86:176-184, 1978.

Konishi T, Hamrick PE, Walsh PJ. Ion transport in the guinea pig cochlea I. Potassium and sodium transport. Acta Otolaryngol. 186:22-34, 1978.

Melichar I, Syka J, Ulehlova L. Recovery of the endocochlear potential and the $\mathrm{K}^{+}$concentrations in the cochlear fluids after acoustic trauma. Hear. Res. 2:55-63, 1980.

Ninoyu O, Meyer zum Gottesberge AM. Changes in $\mathrm{Ca}^{++}$activity and DC potential in experimentally induced endolymphatic hydrops. Arch. Otorhinolaryngol. 243:106-107, 1986.

Salt AN, DeMott JE. Endolymph calcium increases with time after surgical induction of hydrops in guinea pigs. Hear. Res. 74:115121, 1994.

Salt AN, DeMott JE. Endolymph volume changes during osmotic dehydration measured by two marker techniques. Hear. Res. 90:12-23, 1995.

SAlt AN, DeMotт JE. Longitudinal endolymph flow associated with acute volume increase in the guinea pig cochlea. Hear. Res. 107:29-40, 1997.

Salt AN, DeMotт JE. Longitudinal endolymph movements induced by perilymphatic injections. Hear. Res. 123:137-147, 1998.

SAlt AN, Konishi T. Effects of noise on cochlear potential and endolymph potassium concentration recorded with potassiumselective electrodes. Hear. Res. 1:343-363, 1979.

SALt AN, Vora A. Calibration of ion-selective microelectrodes for use with high levels of interfering ions. J. Neurosci. Meth. 28:233-237, 1991.

Salt AN, Thalmann R, Marcus DC, Bohne BA. Direct measurement of longitudinal endolymph flow rate in the guinea pig cochlea. Hear. Res. 23:141-151, 1986.

Salt AN, DeMott JE, Kimura RS. Comparison of endolymph crosssectional area measured histologically with that measured in vivo with an ionic volume marker. Ann. Otol. Rhinol. Laryngol. 104:886-894, 1995.

SEwELL WF. The effects of furosemide on the endocochlear potential and auditory nerve fiber tuning curves in cats. Hear. Res. 14:305-314, 1984.

van Deelen GW, Ruding PR, Veldman Je, Huizing EH, SmoorenBURG GF. Electrocochleographic study of experimentally induced endolymphatic hydrops. Arch. Otorhinolaryngol. 244:167-173, 1987.

Wit HP, Warmerdam TJ, Albers FWJ. Measurement of the mechanical compliance of the endolymphatic compartments of the guinea pig. Hear. Res. 145:82-90, 2000.

Yeh TH, Tsai MC, Lee SY, Hsu MM, Tran Ba Huy P. Stretch-activated nonselective cation, $\mathrm{Cl}^{-}$and $\mathrm{K}^{+}$channels in apical membrane of epithelial cells of Reissner's membrane. Hear. Res. 109:1-10, 1997.

Zidanic M, Brownell WE. Fine structure of the intracochlear electric field I. The silent current. Biophys. J. 57:1253-1268, 1990.

Zidanic M, BRownell WE. Fine structure of the intracochlear potential field II. Tone evoked waveforms and cochlear microphonics. J. Neurophysiol. 67:108-124, 1992. 